\title{
A SHORT NOTE ON THE VOLUME OF HYPERSPHERE*
}

\author{
WOONCHUL $\mathrm{HAM}^{\dagger}$ AND KEMIN ZHOU $\ddagger$
}

\begin{abstract}
In this note, a new method for deriving the volume of hypersphere is proposed by using probability theory. The explicit expression of the multiple times convolution of the probability density functions we should use is very complicated. But in here, we don't need its whole explicit expression. We just need the only a part of information and this fact make it possible to derive the general expression of the voulume of hypersphere. We also comments about the paradox in the hypersphere which was introduced by R.W.Hamming.
\end{abstract}

Key words. Hypersphere, Probability, Convolution, Paradox

1. Introduction. In this note, a new approach to derive the volume of hypersphere is proposed. Even though the volume formula has been already derived and introduced in many books written by G. P. collins 1, and J. H. Conway and N. J. A. Sloane 2], R. W. Hamming [3, the method proposed in this note may be useful for solving many unsolved problems in hyper dimensional space. The proposed method is motivated from the probability theory, especially the mathematical fact that the probability density function of summation of two random variables can be expressed as a convolution of the two probability density functions. We would expect that the explicit expression of the multiple times convolution of the same probability density functions may converges to a Gaussian distribution regardless of its initial shape of probability density function. Also it is very complicated to describe the whole process of this multiple times convolution and to derive the explicit expression of the finite multiple times convolution. Fortunately, only a small part of information, such as those values of the probability functions in the domain between 0 and 1 , is needed in the derivation of volume of hypersphere a nd this fact make it possible to derive the general formula for the hypersphere. We also briefly review the conventional two methods to drive the volume of hyperspere. Instinctive geometric properties of hypersphere is assumed and the gamma integral is used in the conventional approach and these methods may be more concise compared with the new method we propose but instinctive geometric properties are not needed in our approach and this is the advantage of our approach. We also comment on the paradox introduced in [3].

2. Conventional Method. In this section, we briefly review the conventional methods for deriving the formula of the volume of hypershpere.

2.1. Method A. At first, we review the method introduced by Hamming [3]. He used the following well known property of gamma integral.

$$
[\Gamma(1 / 2)]^{n}=\pi^{n / 2}=\int_{\infty}^{\infty} \int_{\infty}^{\infty} \cdots \int_{\infty}^{\infty} e^{-r^{2}} d x_{1} d x_{2} \cdots d x_{n}
$$

where

$$
r^{2}=x_{1}^{2}+x_{2}^{2}+\cdots+x_{n}^{2}
$$

${ }^{*}$ This work was completed with the partial support of Mechatronic Research center of Chonbuk National University

†Woonchul Ham is with the Electronics and Information Division, Chonbuk National University, Chonju-si, Chonbuk, South Korea(wcham@moak.chonbuk.ac.kr) and this work is completed while he is a visiting professor at Louisiana State University.

${ }^{\ddagger}$ Kemin Zhou is with the Department of Electrical and Computer Engineering, Louisiana State University, Baton Rouge, LA 70803, USA (kemin@ece.1su.edu). 
He also assume that the volume $V_{n}(r)$ of an $n$-dimensional sphere depends on the radius $s$ as $r^{n}$ by reflection on the classical Euclidian geometry as follows.

$$
V_{n}(r)=C_{n} r^{n}
$$

He proposed the following equation by using the geometric instinct.

$$
\int_{\infty}^{\infty} \int_{\infty}^{\infty} \cdots \int_{\infty}^{\infty} e^{-r^{2}} d x_{1} d x_{2} \cdots d x_{n}=\int_{0}^{\infty} e^{-r^{2}} \frac{d V_{n}(r)}{d r} d r
$$

From the above equations, we have

$$
C_{n}=\frac{\pi^{n / 2}}{\Gamma\left(\frac{n}{2}+1\right)}
$$

2.2. Method B. Next, we review the method introduced by G. P. collins [1]. In here, $S^{n}$ denote the hypersurface of $n$ - dimensional hypersphere.

The volume contained inside $S^{n}$ is given by the integral

$$
V_{n}(r)=\int_{\sum_{i=1}^{n} x_{i}^{2} \leq r^{2}} d x_{1} d x_{2} \cdots d x_{n}
$$

Going to polar coordinates $\left(r^{2}=\sum_{i=1}^{n} x_{i}^{2}\right)$ this becomes

$$
V_{n}(r)=\int_{S^{n}} d \Omega \int_{0}^{r} r^{n-1} d r
$$

The first integral is the integral over all solid angles subtended by the sphere and is equal to its area $A(n)=\frac{2 \pi^{n / 2}}{\Gamma\left(\frac{n}{2}\right)}$. The second integral is elementary and evaluates to $\int_{0}^{r} r^{n-1} d r=r^{n} / n$.

Finally, the volume is

$$
V_{n}(r)=\frac{\pi^{n / 2}}{\frac{n}{2} \Gamma\left(\frac{n}{2}\right)} r^{n}=\frac{\pi^{n / 2}}{\Gamma\left(\frac{n}{2}+1\right)} r^{n} .
$$

Note that this formula works for $n \geq 0$. The first few cases are

- $n=2: \Gamma(2)=1$, hence $V_{2}(1)=\pi$ (this is the familiar result for the area of the unit circle);

- $n=3: \Gamma(5 / 2)=3 \sqrt{\pi} / 4$, hence $V_{3}(1)=4 \pi / 3$ (this is the familiar result for the volume of the unit sphere);

- $n=4: \Gamma(3)=2$, hence $V_{4}(1)=\pi^{2} / 2$.

3. Mathematical Preliminary. We briefly introduce the convolution theory in random variables which will be used in the next section, see [4]

THEOREM 3.1. The probability density function $p_{z}(z)$ of the summation of several random variables, such as, $z=x_{1}+x_{2}+\cdots+x_{n}$ is expressed as follows

$$
p_{z}(z)=p_{x_{1}}(z) * p_{x_{2}}(z) * \cdots * p_{x_{n}}(z),
$$


where operator $*$ denotes the convolution of functions.

THEOREM 3.2. Let the probability density function of $x$ be uniformly distributed between -1 and 1. Then, the probability density function of random variable $x^{2}, p_{x^{2}}(z)$, is described by

$$
p_{x^{2}}(z)= \begin{cases}0 & \text { if } z<0 \\ \frac{1}{2} z^{-1 / 2} & \text { if } 0 \leq z \leq 1 \\ 0 & \text { otherwise }\end{cases}
$$

Let us define the following probability density functions:

$$
\begin{gathered}
p_{1}(z)=p_{x^{2}}(z) \\
p_{2}(z)=p_{1}(z) * p_{1}(z) \\
p_{3}(z)=p_{1}(z) * p_{1}(z) * p_{1}(z) \\
\cdots \\
p_{n}(z)=\underbrace{p_{1}(z) * \cdots * p_{1}(z)}_{\mathrm{n}}
\end{gathered}
$$

The complete explicit expression of the above probability density function may converges to some types of Gaussian distribution function. Therefore, it is a very difficult problem to find the explicit forms of above probability density functions for finite $n$. But only the first part of information, i.e., explicit form of above probability density functions in the domain of $z$ between 0 and 1 , is needed in the derivation of the volume of hypersphere. Because of this fact, we will only derive the explicit form of each probability function in the domain $z$ between 0 and 1 in the next section

4. Probability Density Functions. In this section, we want to derive the explicit form in the domain of $z$ between 0 and 1 for each probability function $p_{i} n(z)$ defined in the previous section. From the mathematical preliminary, the probability density function $p_{2}(z)=p_{1}(z) * p_{1}(z)$ and it is expressed as follows.

$$
p_{2}(z)= \begin{cases}\int_{0}^{z} \frac{1}{4} \lambda^{-1 / 2}(z-\lambda)^{-1 / 2} d \lambda & \text { if } 0 \leq z \leq 1 \\ \int_{z-1}^{1} \frac{1}{4} \lambda^{-1 / 2}(z-\lambda)^{-1 / 2} d \lambda & \text { if } 1 \leq z \leq 2 \\ 0 & \text { otherwise }\end{cases}
$$

The above equation can be simplified by calculating the integration as follows.

$$
p_{2}(z)= \begin{cases}\frac{\pi}{4} & \text { if } 0 \leq z \leq 1 \\ \frac{1}{2} \sin ^{-1}\left(\frac{1-z / 2}{z / 2}\right) & \text { if } 1 \leq z \leq 2 \\ 0 & \text { otherwise }\end{cases}
$$

Now, we derive the first part, that is, in the domain of $z$ between 0 and 1 , of the probability density function $p_{3}(z)$ by using convolution of $p_{2}(z) * p_{1}(z)$. It is easy to see that the first part of $p_{3}(z)$ can be expressed as follows. 


$$
p_{3}(z)= \begin{cases}\frac{\pi}{8} \int_{0}^{z}(z-\lambda)^{-1 / 2} d \lambda & \text { if } 0 \leq z \leq 1 \\ \text { not needed } & \text { otherwise }\end{cases}
$$

It is easy to obtain the following simplified equation for the first part of $p_{3}(z)$,

$$
p_{3}(z)= \begin{cases}\frac{\pi}{4} z^{1 / 2} & \text { if } 0 \leq z \leq 1 \\ \text { not needed } & \text { otherwise }\end{cases}
$$

The important thing to note in derivation of the above equations is that we only use the function shape of $p_{1}$ and $p_{2}$ in the domain $z$ between 0 and 1 to derive the explicit expression of function shape of $p_{3}$ in the domain $z$ between 0 and 1 . The function shape of $p_{1}$ and $p_{2}$ in the domain $z$ between 0 and 1 is simple and this

Now we can derive the general form of the first part, that is, in the domain $z$ between 0 and 1, of the probability density function when $n$ is even, such as $n=2 m$, by using the multiple times convolution of $p_{2}(z)$ as follows:

$$
p_{2 m}(z)= \begin{cases}\underbrace{p_{2}(z) * \cdots * p_{2}(z)}_{m} & \text { if } 0 \leq z \leq 1 \\ \text { not needed } & \text { otherwise }\end{cases}
$$

It is easy to obtain the following simplified equation for the first part of $p_{2 m+2}(z)$ when $m \geq 1$,

$$
p_{2 m+2}(z)= \begin{cases}\left(\frac{\pi}{4}\right)^{m+1} \frac{z^{m}}{(m) !} & \text { if } 0 \leq z \leq 1 \\ \text { not needed } & \text { otherwise }\end{cases}
$$

Next, we derive the general form of the first part of the probability density function when $n$ is odd, that is when $n=2 m+2+1$, by using convolution of $p_{2 m+2}(z) * p_{1}(z)$ as follows:

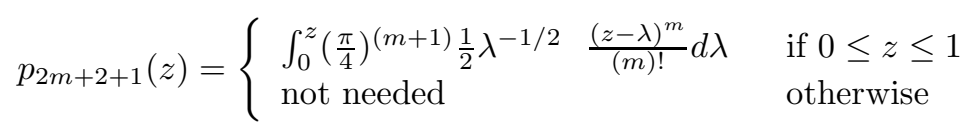

By using several partial integrations, we can obtain the following simplified form for $p_{2 m+2+1}(z)$.

$$
p_{2 m+2+1}(z)= \begin{cases}\left(\frac{\pi}{4}\right)^{(m+1)} \frac{2}{3} \frac{2}{5} \frac{2}{7} \cdots \frac{2}{2 m+1} z^{\frac{2 m+1}{2}} & \text { if } 0 \leq z \leq 1 \\ \text { not needed } & \text { otherwise }\end{cases}
$$

where $m \geq 1$.

5. Volume Formula of Hypersphere. In this section, we propose a new method to derive the volume of $n$-dimensional unit hypersphere. Let us consider the $n$ - dimensional hypercube and assume that the length of each edge of this hypercube is 2 . Let us think about choosing a point in this hypercube. Now we want to calculate the probability $p_{\text {hyper }}$ that the point chosen as above is belonging to the $n$-dimensional unit hypersphere contained in the above hypercube. We can easily see 
that the probability $p_{\text {hyper }}$ is the ratio between the hyper volume $V_{s n}$ of unit hypersphere and hyper volume $V_{c n}$ of the hypercube defined above and it can be expressed as follows:

$$
\begin{gathered}
p_{\text {hyper }}=\frac{\text { the volume of } V_{s n}}{\text { the volume of } V_{c n}} \\
=\frac{\text { Volume of }\left\{\left(x_{1}, x_{2}, \cdots, x_{n}\right) \in \mathbb{R}^{n}: x_{1}^{2}+x_{2}^{2}+\cdots+x_{n}^{2}<1\right\}}{\text { Volume of }\left\{\left(x_{1}, x_{2}, \cdots, x_{n}\right) \in \mathbb{R}^{n}:-1 \leq x_{1} \leq 1,-1 \leq x_{2} \leq 1, \cdots,-1 \leq x_{n} \leq 1\right\}},
\end{gathered}
$$

where $V_{c n}=2^{n}$. Now, we propose a way to calculate the volume of a hypersphere.

THEOREM 5.1. The volume of $n$-dimensional hypersphere $V_{s n}$ is expressed as

$$
V_{s n}=C_{n} r^{n},
$$

where $r$ denotes the radius of hypersphere and $C_{n}$ denotes the volume of a unit hypersphere,

$$
C_{n}=2^{n} \cdot p_{\text {hyper }}
$$

and

$$
p_{\text {hyper }}=\int_{0}^{1} p_{n}(z) d z .
$$

It is clear from the above theorem, we only need to integrate the probability density function in the domain of $z$ between 0 and 1 . From the property of convolution, we only need the information of probability density functions in the domain of $z$ between 0 and 1 to derive the same part of information of the convolution.

It is clear that

$$
C_{n}= \begin{cases}\left(\frac{\pi^{n / 2}}{(n / 2) !}\right) & \text { if } n \text { is even } \\ \frac{\pi^{(n-1) / 2} 2^{(n+1) / 2}}{3 \cdot 5 \cdot 7 \cdots n} & \text { if } n \text { is odd }\end{cases}
$$

where $n \geq 2$.

Therefore, we finally obtain the general form of volume for hypersphere as follows:

$$
V_{n}(r)= \begin{cases}\left(\frac{\pi^{n / 2}}{(n / 2) !}\right) r^{n} & \text { if } n \text { is even } \\ \frac{\pi^{(n-1) / 2} 2^{(n+1) / 2}}{3 \cdot 5 \cdot 7 \cdots n} r^{n} & \text { if } n \text { is odd }\end{cases}
$$

where $n \geq 2$. Therefore if we we the gamma function, the volumes of $n-$ dimensional hypershpere is expressed as

$$
V_{n}(r)=\frac{\pi^{n / 2}}{\Gamma\left(\frac{n}{2}+1\right)} r^{n} .
$$

The volumes of hypershpere in 2,3,4,5 and 6 dimensional cases are shown in the table 1 and we can see that the above formula is the same as conventinal one introduced in the previous section. 
6. Paradox. In this section, we comment on the paradox in the hypersphere introduced by Hamming [3]. He considered a $4 \times 4 \times \cdots \times 4$ cube and suggested that there exists an inner sphere whose radius is greater than 2 and this inner sphere will reaches the outside cube! at $n=10$, where $n$ is the order of dimension of the hypercube. But if we consider more deeply, then we can see that this thinking is wrong and this kind of paradox is due to the fact that we can not exactly catch the geometry of hypercube. It is sure that there exists a inner sphere whose radius is greater than 2 but it never reaches the outside of hypercube at $n=10$. Let us think about the longest distance $l_{\max }$ between two vertexes in this hypercube. We can easily see that $l_{\max }=4 \sqrt{10}$ and this value is unexpectedly large. So we can see that there is enough space in a hypersphere which can contain inner sphere whose radius is greater than 2 . One more important fact in a $n$ - dimensional hypercube is that the outer hyprespheres such as those considered in [3] cover only a small part of hypercube near the surface and there is still a huge space within the hypercube uncovered by the outer hyperspheres. Can you imagine it?

7. Conclusion. In this short note, a new method to derive the volume of hypersphere is proposed and the approach is very different from conventional geometric approach. In this approach, we only use the properties of probability density function, especially the convolution properties. In this approach, we do not use any geometric properties of hyper dimensional space and this may be an advantage of this approach. The approach introduced in this short note may be helpful for the researchers who are interested in unsolved problems in the research area of hyper dimensional space, such as hyper packing problem and hyper covering in information theory.

\section{REFERENCES}

[1] G. P. Collins, " The Shapes of Space, " Sci. Amer. 291, July, 2004.

[2] J. H. Conway And N. J. A. Slonne, Sphere Packings, Lattices, and Groups, New York:Springer-Verlag, 1993

[3] R. W. Hamming, Coding and Information Theory, Prentice-Hall, 1980.

[4] A. Papoulis, Probabilty, Random Variables, and Stochastic Processes, McGraw-Hill, 2002

TABLE 5.1

Table of the Volume for Hypershpheres

\begin{tabular}{|c|c|c|}
\hline$n$ & $C_{n}$ & $V_{n}$ \\
\hline \hline 2 & $\pi$ & $\pi r^{2}$ \\
\hline 3 & $4 \pi / 3$ & $4 \pi / 3 r^{3}$ \\
\hline 4 & $\pi^{2} / 2$ & $\pi^{2} / 2 r^{4}$ \\
\hline 5 & $8 \pi^{2} / 15$ & $8 \pi^{2} / 15 r^{5}$ \\
\hline 6 & $\pi^{3} / 6$ & $\pi^{3} / 6 r^{6}$ \\
\hline
\end{tabular}

